\title{
TEORES DE ISOFLAVONAS E CAPACIDADE ANTIOXIDANTE DA SOJA E PRODUTOS DERIVADOS ${ }^{1}$
}

\author{
Ana Cristina Lopes BARBOSA², Neuza Mariko Aymoto HASSIMOTTO², \\ Franco Maria LAJOLO ${ }^{2}$, Maria Inés GENOVESE ${ }^{2, *}$
}

\begin{abstract}
RESUMO
O objetivo deste estudo foi determinar a capacidade antioxidante, o teor e as formas de isoflavonas presentes nos produtos derivados de soja e compará-los à soja em grão. A capacidade antioxidante foi mensurada por meio do método de cooxidação do $\beta$-caroteno/ ácido linoléico e pelo seqüestro de radicais livres ( $\mathrm{DPPH} \bullet$ ). Os resultados mostraram que o conteúdo de isoflavonas nos produtos variou de 95 a $711 \mathrm{mg} / 100 \mathrm{~g}$ (b.s.) e o teor de fenólicos totais de 101 a $413 \mathrm{mg}$ equivalentes de catequina/100 g (b.s.), sendo que o grão de soja apresentou um conteúdo de 110 e $200 \mathrm{mg} / 100 \mathrm{~g}$ (b.s.), respectivamente, para isoflavonas e fenólicos totais. Em relação às formas de isoflavonas a farinha integral e sua respectiva farinha desengordurada e a farinha desengordurada de soja comercial apresentaram predominância de conjugados malonilglicosídeos e $\beta$-glicosídeos, similar aos grãos de soja. O suplemento à base de gérmen de soja e a farinha desengordurada de soja apresentaram a maior capacidade antioxidante por ambos os métodos (5 $\mu$ moles equivalentes de trolox/g e $\sim 0,8 \mu$ moles equivalentes de BHT/g amostra b.s.). Os resultados demonstraram que o conteúdo total e tipo de isoflavonas e o teor de fenólicos dos produtos de soja dependem do processamento e que a capacidade antioxidante desses produtos varia significativamente.
\end{abstract}

Palavras-chave: soja e derivados protéicos, isoflavonas, capacidade antioxidante.

\section{SUMMARY}

ISOFLAVONE CONTENT AND PROFILE AND ANTIOXIDANT ACTIVITY OF SOY AND SOY PRODUCTS. The objective of this study was to determine the antioxidant activity and isoflavone content and type of soy seeds and soy products. The antioxidant activity was measured using $\beta$-carotene bleaching and 2.2-diphenyl-1-picryl-hydrazyl (DPPH •) free radical methods. The total isoflavone content of soy products varied from 95 to $711 \mathrm{mg} / 100 \mathrm{~g} \mathrm{DW}$ (expressed as aglycones) and total phenolics content from 101 to $413 \mathrm{mg}$ equivalents of catechin/100 g DW. Soybeans presented 110 and $200 \mathrm{mg} / 100 \mathrm{~g} \mathrm{DW}$ of isoflavones and total phenolics, respectively. The $\beta$-glycosides and malonylglycosides were the main forms of the isoflavones found in the whole flour, defatted flour and commercial defatted flour, similar to the soy seeds. The soy germ (hypocotyl) and the defatted soy flours presented the highest antioxidant activities in both methods (5.0 $\mu$ moles equivalents of trolox/g DW and $\sim 0.8 \mu$ moles equivalents of BHT/g DW). Taking this into account, the results showed that total isoflavone and phenolic contents and isoflavone profile of soy products depend on processing conditions and that antioxidant activity varied significantly among products.

Keywoards: soy, soy products, isoflavones, antioxidant activity.

\section{1 - INTRODUÇÃO}

Os alimentos de origem vegetal apresentam compostos não-nutrientes (fitoquímicos) com atividades biológicas ditas promotoras da saúde, tais como atividades antioxidante, antiinflamatória e hipocolesterolêmica. Entre estes podemos citar as catequinas do chá verde e do vinho, as antocianinas dos frutos vermelhos, os flavonóis das folhas e as isoflavonas da soja [12].

Os seres humanos estão expostos a uma série de agentes oxidantes que podem causar danos oxidativos a biomoléculas (lipídeos, proteínas e ácidos nucléicos) que poderiam levar a um grande número de patologias incluindo câncer

\footnotetext{
${ }^{1}$ Recebido para publicação em 9/4/2006. Aceito para publicação em 20/10/2006 (001727)

${ }^{2}$ Laboratório de Química e Bioquímica de Alimentos, Departamento de Alimentos e Nutrição Experimental,

Faculdade de Ciências Farmacêuticas, Universidade de São Paulo (USP)

Av. Prof. Lineu Prestes, 580, Bloco 14, CEP 05508-900,

São Paulo (SP), Brasil,

E-mail: genovese@usp.br

* A quem a correspondência deve ser enviada
}

e arteriosclerose. Assim, presume-se que a ingestão de antioxidantes capazes de neutralizar os radicais livres possa ter um papel importante na prevenção destas doenças [12, 7]. Dessa forma, a identificação de fontes vegetais com alta capacidade antioxidante é de extrema importância.

Ainda, a possibilidade de prevenir e/ou combater doenças por meio da dieta tem atraído a atenção, tanto da comunidade científica como das indústrias alimentícias, com o objetivo comum de desenvolver os atualmente conhecidos como "alimentos funcionais" ou alimentos ricos em um ou mais compostos/componentes bioativos que apresentam efeitos positivos na saúde.

A soja e seus derivados apresentam teores variáveis de isoflavonas (daidzeína, genisteína e gliciteína), compostos bioativos com diversas atividades biológicas, as quais parecem estar relacionadas com as suas formas [16]. As isoflavonas apresentam-se em 4 formas químicas, somando assim 12 isômeros: as agliconas daidzeína, genisteína e gliciteína; os $\beta$-glicosídeos daidzina, genistina e glicitina; e os derivados glicosilados acetilados 6"-O-acetildaidzina, 6"-O-acetilgenistina, 6"-O-acetilglicitina; e glicosilados malonilados 6"-O-malonildaidzina, 6"-O-malonilgenistina e 6"-O- malonilglicitina [14, 11]. 
Os derivados protéicos da soja, como farinhas desengorduradas, isolados, concentrados e texturizados protéicos são amplamente utilizados na indústria alimentícia em decorrência de suas propriedades funcionais. Esses produtos contêm quantidades apreciáveis de isoflavonas, as quais têm mostrado em dados experimentais e clínicos que representam uma alternativa promissora na prevenção e/ou tratamento de muitas doenças hormônio-dependentes, incluindo câncer, sintomas da menopausa, doenças cardiovasculares e osteoporose [26, 21, 29]. Durante as etapas do processamento desses produtos da soja pode haver perda de algumas isoflavonas e também mudança no seu perfil. As principais isoflavonas presentes na soja não-processada, malonilgenistina, genistina, malonildaidzina e daidzina, são transformadas para outras formas durante o processamento, tais como acetilglicosídeos e agliconas [27].

A capacidade antioxidante das isoflavonas foi relacionada ao número de grupos hidroxila presente na sua estrutura química. NAIM et al. [18] reportaram que a capacidade antioxidante das isoflavonas decresce com a glicosilação ou a substituição do grupo hidroxila pelo grupo metoxila. As isoflavonas podem inibir a peroxidação lipídica in vitro por ação de seqüestro de radicais livres ou por atuar como agentes quelantes de metais [4]. PRATT \& BIRAT [19] reportaram que farinha desengordurada, concentrado e isolado protéicos de soja apresentaram atividade antioxidante significativa pelo método de cooxidação do $\beta$-caroteno/ácido linoléico. Já entre as três agliconas, genisteína apresentou a maior capacidade antioxidante, determinada pelo mesmo sistema [20].

Recentemente, YEN \& LAI [28] reportaram que a suplementação com isoflavonas purificadas (daidzeína e genisteína) ou extrato metanólico $80 \%$ obtido a partir de alguns produtos de soja, tais como tofu, inibiu a oxidação de espécies de nitrogênio reativo in vitro e in vivo. A inibição foi correlacionada positivamente com o conteúdo total de isoflavonas nos extratos.

O desenvolvimento de produtos alimentícios com teores apreciáveis de isoflavonas e, portanto capacidade antioxidante, requer o conhecimento do conteúdo presente nos diversos derivados protéicos de soja e o efeito do processamento. Para tanto, o objetivo deste estudo foi determinar o teor e as formas de isoflavonas e a capacidade antioxidante dos produtos derivados de soja (farinhas, isolados, texturizados, e suplemento comercial à base de gérmen de soja) e compará-los à soja em grão.

\section{2 - MATERIAL E MÉTODOS}

\section{1 - Material}

Soja em grão e seis derivados (3 lotes de cada produto): farinha desengordurada de soja comercial, farinha integral e sua respectiva farinha desengordurada (obtida em laboratório utilizando-se n-hexano como solvente), isolado protéico de soja, proteína texturizada de soja e um suplemento comercial de isoflavonas (à base de gérmen de soja) foram obtidos no comércio local e da Exin Internacional Ltda (São Paulo, SP). O conteúdo de 60 cápsulas do suplemento (proveniente de 3 amostras) foi homogeneizado e passado em peneira 60 mesh $(0,25 \mathrm{~mm})$. Todas as outras amostras foram moídas em moinho Janke \& Kunkel A-10 (Ika \& Works, Inc., Wilmington, E.U.A.) e passadas em peneira 60 mesh $(0,25 \mathrm{~mm})$ previamente à extração. Todos os reagentes e solventes utilizados foram de grau analítico ou para cromatografia líquida.

\section{2 - Métodos}

\subsection{1 - Extração das isoflavonas}

A extração das isoflavonas foi realizada com agitador mecânico e barra magnética por $2 \mathrm{hs} \mathrm{a} 4{ }^{\circ} \mathrm{C}$, na proporção de 1:20 (m/v), com metanol 80\% [8]. Os extratos foram filtrados utilizando-se papel de filtro Whatman $n^{\circ} 6$ e submetidos à rotaevaporação (Rotavapor ${ }^{\circledR}$ RE 120 - Büchi, Flawil, Suíça) até atingir o volume de $2 \mathrm{~mL}$. A seguir completou-se o volume para $5 \mathrm{~mL}$ em balão volumétrico com metanol (grau HPLC). As amostras foram filtradas utilizando-se filtros de polietileno com membrana PTFE (Millipore Ltd., Bedford, E.U.A.) de 0,45 $\mu \mathrm{m}$ de poro para posterior análise por CLAE. As extrações foram realizadas em triplicata.

\subsection{2 - Cromatografia líquida de alta eficiência}

A separação das isoflavonas foi realizada em coluna $\mathrm{C}$ $18 \mu$ Bondapak (Waters, Milford, E.U.A.) de acordo com o método de SONG et al. [25]. O cromatógrafo líquido utilizado foi o da Hewlett Packard (Palo Alto, E.U.A.) série 1100, equipado com injetor automático de amostras, bomba quaternária e detetor com arranjo de diodos, controlados pelo software ChemStation. Os padrões de daidzeína e genisteína foram obtidos da Sigma Chemicals Co. (St. Louis, E.U.A.), daidzina e genistina foram obtidos da Apin Chemicals Ltd. (Abingdon, Reino Unido), glicitina e gliciteína foram obtidos da Fujicco Co. Ltd. (Kyoto, Japão), acetildaidzina, acetilgenistina, acetilglicitina, malonildaidzina, malonilgenistina e malonilglicitina foram obtidos da LC Laboratories (Woburn, E.U.A.). A identificação foi feita a partir dos tempos de retenção e dos espectros. As amostras foram injetadas em duplicata. Os resultados foram expressos na forma de média \pm desvio-padrão, como mg de isoflavonas (agliconas) por $100 \mathrm{~g}$ de amostra (b.s.). As formas das isoflavonas ( $\beta$-glicosídeos, malonilglicosídeos, acetilglicosídeos e agliconas) foram expressas em\% do total (p/p).

\subsection{3 - Determinação de fenólicos totais}

O conteúdo de fenólicos totais foi determinado de acordo com o método de ZIELISKI \& KOZOWSKA [30], com algumas modificações [10], descritas a seguir. Alíquotas de 0,25 mL dos extratos metanólicos $80 \%$ (triplicata) foram adicionadas de $2 \mathrm{~mL}$ de água destilada e 0,25 mL do reagente Folin-Ciocalteau. Após 3 min à temperatura ambiente, foi adicionado 
0,25 mL de solução saturada de carbonato de sódio. Os tubos foram levados para o banho-maria a $37^{\circ} \mathrm{C}$ por $30 \mathrm{~min}$ para desenvolver a coloração. Solução de catequina $(0,2 \mathrm{mg} / \mathrm{mL}$ metanol) foi utilizada como padrão. As leituras de absorbância foram realizadas no espectrofotômetro Hewlett-Packard 8453 (Palo Alto, E.U.A.). Os resultados foram expressos como mg de catequina por $100 \mathrm{~g}$ de amostra (b.s.). As análises foram realizadas em triplicata.

\subsection{4 - Determinação da capacidade antioxidante - seqüestro de radicais livres do DPPH・}

A capacidade antioxidante foi determinada pela redução do radical estável DPPH • (2,2-difenil-1-picrilhidrazil) pelos antioxidantes presentes na amostra, segundo descrito por BRAND-WILLIAMS, CUVELIER \& BERSET [3]. A solução de DPPH (Sigma Chemical Co., St. Louis, E.U.A.), $\sim 20 \mathrm{mg} / \mathrm{mL}$ em metanol, foi preparada de forma a apresentar absorbância em $517 \mathrm{~nm}$ entre 0,6 e 0,7. As determinações foram realizadas em microplaca de poliestireno com 96 cavidades (Costar, Cambrigde, E.U.A.) para uso em comprimento de onda entre 340 e $800 \mathrm{~nm}$. Em cada cavidade da microplaca, foram adicionados $250 \mu \mathrm{L}$ da solução de DPPH •, $40 \mu \mathrm{L}$ de metanol para o grupo controle ou o mesmo volume de extrato para as amostras, adequadamente diluídas. A reação foi mantida à temperatura ambiente por $20 \mathrm{~min}$, no escuro. As leituras das absorbâncias foram realizadas em $517 \mathrm{~nm}$ em espectrofotômetro de microplaca Benchmark Plus (Bio-Rad Laboratories, Hercules, E.U.A.). A absorbância das amostras $(\mathrm{Am})$ correlacionada à absorbância do controle (Ac) resulta na porcentagem de seqüestro de radicais livres (\% SRL), que pode ser expressa pela seguinte fórmula:

$\% S R L=\left(\frac{A c-A m}{A c}\right) \times 100$

A curva de calibração foi preparada com uma solução de Trolox (Sigma Chemical Co., St. Louis, E.U.A.), e os resultados foram expressos em $\mu$ moles equivalentes de Trolox/g de amostra (b.s.). As análises foram realizadas em triplicata.

\subsection{5 - Determinação da capacidade antioxidante - sistema co-oxidação do $\beta$-caroteno/ácido linoléico}

A capacidade antioxidante foi avaliada pelo método de descoramento do $\beta$-caroteno [17], com pequenas modificações [13], descritas a seguir. Para o preparo da solução reativa, alíquotas de $20 \mu \mathrm{L}$ de uma solução de $\beta$-caroteno em clorofórmio ( $2 \mathrm{mg} / \mathrm{mL}$ ) foram misturadas com $30 \mu \mathrm{L}$ de ácido linoléico, $1 \mathrm{~mL}$ de clorofórmio e $200 \mu \mathrm{L}$ de Tween 40. Posteriormente, o clorofórmio foi evaporado até secagem completa, sob nitrogênio. Após evaporação do clorofórmio, foram adicionados aproximadamente $30 \mathrm{~mL}$ de água destilada saturada de oxigênio e a mistura foi agitada. A absorbância da solução foi então ajustada com água para 0,6. Para a reação de oxidação, uma alíquota $(9 \mu \mathrm{L})$ do extrato foi adicionada a $241 \mu \mathrm{L}$ da solução de $\beta$-caroteno em placa de microtitulação. As amostras foram então submetidas à auto-oxidação a $45{ }^{\circ} \mathrm{C}$ for $3 \mathrm{~h}$. A absorbância da solução a $470 \eta \mathrm{m}$ foi medida no tempo zero e em intervalos de $15 \mathrm{~min}$, usando-se o Microplate Spectrophotometer Benchmark Plus (Bio-Rad Laboratories, Hercules, E.U.A.). Para o controle, foi utilizado metanol. As análises foram realizadas em triplicata. A capacidade antioxidante foi calculada pela porcentagem de inibição relativa ao controle (100\% de oxidação).

\subsection{6 - Umidade}

A umidade da soja em grão, farinha desengordurada comercial, farinha integral, farinha desengordurada, isolado protéico, proteína texturizada e suplemento à base de gérmen de soja foi determinada por dessecação em estufa a $105^{\circ} \mathrm{C}$ até peso constante [1]. As análises foram realizadas em triplicata.

\subsection{7 - Análise estatística dos resultados}

Os resultados foram expressos na forma de média \pm desvio-padrão $(\mathrm{n}=3$ ). Para a análise estatística dos resultados utilizou-se o programa Statistica versão 5.0 da StatSoft (Tulsa, E.U.A.). A comparação das médias foi realizada por análise de variância (ANOVA) e a seguir pelo teste LSD $(\mathrm{p}<0,05)$.

\section{3 - RESULTADOS E DISCUSSÃO}

Os resultados mostraram que o conteúdo de isoflavonas nos produtos variou de 95 a $711 \mathrm{mg} / 100 \mathrm{~g}$ (b.s.) e o teor de fenólicos totais de 101 a $413 \mathrm{mg}$ equivalentes de catequina/100 g (b.s.), sendo que o grão de soja apresentou um conteúdo de 110 e 200 mg/100 g (b.s.), respectivamente, para isoflavonas e fenólicos totais e o suplemento à base de gérmen de soja, os maiores teores de ambos (Tabela 1). Além disso, foi observada uma alta correlação linear entre estas duas variáveis $(\mathrm{r}=0,94)$.

TABELA 1 - Teores de umidade (\%), de fenólicos totais e de isoflavonas (agliconas) totais (mg/100 g de amostra b.s.) da soja em grão, farinha desengordurada comercial, farinha integral, farinha desengordurada, isolado protéico, proteína texturizada e suplemento à base de gérmen de soja.

\begin{tabular}{lccc}
\hline \multicolumn{1}{c}{ Amostras } & Umidade & $\begin{array}{c}\text { Fenólicos } \\
\text { totais }\end{array}$ & $\begin{array}{c}\text { Isoflavonas } \\
\text { totais }\end{array}$ \\
\hline $\begin{array}{l}\text { Farinha desengordurada } \\
\text { de soja comercial }\end{array}$ & $10,5 \pm 0,2^{\mathrm{a}}$ & $200 \pm 10^{\mathrm{c}}$ & $191 \pm 6^{\mathrm{d}}$ \\
$\begin{array}{l}\text { Farinha integral } \\
\text { Farinha desengordurada }\end{array}$ & $\begin{array}{c}10,34 \pm 0,03^{\mathrm{e}} \\
\text { de soja }\end{array}$ & $183 \pm 1^{\mathrm{c}}$ & $200 \pm 1^{\mathrm{c}}$ \\
$\begin{array}{l}\text { Isolado protéico de soja } \\
\text { Proteína texturizada de }\end{array}$ & $4,69 \pm 0,06^{\mathrm{d}}$ & $101 \pm 3^{\mathrm{e}}$ & $124 \pm 1^{\mathrm{e}}$ \\
soja & $6,7 \pm 0,3^{\mathrm{c}}$ & $148 \pm 6^{\mathrm{d}}$ & $95 \pm 7^{\mathrm{g}}$ \\
Grãos de soja & $10,8 \pm 0,3^{\mathrm{a}}$ & $200 \pm 1^{\mathrm{c}}$ & $110 \pm 2^{\mathrm{f}}$ \\
Suplemento & $8,0 \pm 0,3^{\mathrm{b}}$ & $413 \pm 4^{\mathrm{a}}$ & $711 \pm 5^{\mathrm{a}}$ \\
\hline
\end{tabular}

Os resultados estão expressos na forma de média \pm desvio padrão $(n=3)$. Médias na mesma coluna com letras diferentes são significativamente diferentes $(\mathrm{p}<0,05)$. 
A Tabela 2 apresenta as formas de isoflavonas nos diferentes produtos (soja em grão, farinha desengordurada comercial, farinha integral, farinha desengordurada, isolado protéico, proteína texturizada e suplemento à base de gérmen de soja).

A farinha integral, sua respectiva farinha desengordurada e a farinha de soja desengordurada comercial apresentaram predominância de malonilglicosídeos e $\beta$-glicosídeos ( $90 \%$ do total), o que caracteriza os produtos de soja minimamente processados, cujo perfil é similar ao dos grãos de soja [5]. Já a proteína texturizada apresentou um elevado conteúdo de acetilglicosídeos (17\% do total), cuja formação está associada ao processo de extrusão (calor seco), que promove a descarboxilação dos malonilglicosídeos originando os acetilglicosídeos. O isolado protéico apresentou como forma predominante as agliconas (37\% do total), resultado da ação das $\beta$-glicosidases da soja durante o processo de extração, que hidrolisam os $\beta$-glicosídeos transformandoos em agliconas [2]. O suplemento à base de gérmen de soja apresentou quase que exclusivamente $\beta$-glicosídeos (79\% do total). Os derivados de genisteína, seguidos pelos de daidzeína, predominaram em todos os produtos, com exceção do suplemento à base de gérmen de soja, no qual os derivados de genisteína e gliciteína se apresentaram na mesma proporção. Como previamente relatado, o cotilédone se diferencia do hipocótilo (gérmen) de soja, tanto pelos teores mais elevados de isoflavonas presentes neste último como pela diferente proporção em que os derivados de gliciteína, genisteína e daidzeína se apresentam [6].

A Tabela 3 apresenta os valores da capacidade antioxidante determinada pelo método de seqüestro de radicais livres do $\mathrm{DPPH}$ • e pelo sistema $\beta$-caroteno/ácido linoléico da soja em grão e seus derivados.

A capacidade antioxidante da soja e dos seus derivados determinada pelo método do DPPH • variou de 1,3 a $5,1 \mu$ moles equivalentes de Trolox/g de amostra (b.s.), e pelo sistema $\beta$-caroteno/ácido linoléico de 0,58 a 0,85 $\mu$ moles equivalentes de BHT/g de amostra (b.s.) (Tabela 3). O suplemento à base de gérmen de soja e a farinha desengordurada de soja apresentaram a maior capacidade antioxidante em ambos os métodos $(5,1$ e 5,0 $\mu$ moles equivalentes de Trolox/g, respectivamente, e 0,78 e 0,85 $\mu$ moles equivalentes de $\mathrm{BHT} / \mathrm{g}$, respectivamente), e o isolado protéico de soja a menor ( $1,3 \mu$ moles equivalentes de Trolox/g e 0,58 $\mu$ moles equivalentes de $\mathrm{BHT} / \mathrm{g}$ ). O método $\mathrm{DPPH} \bullet$ se baseia na redução deste radical pela abstração do $\mathrm{H} \bullet$ pelo antioxidante, e o método $\beta$-caroteno se baseia na proteção de um substrato lipídico contra os produtos de degradação oxidativa de um ácido graxo [24]. Observou-se uma alta correlação entre os valores obtidos por meio dos dois métodos $(\mathrm{r}=0,93)$, o que é esperado, uma vez que tanto o método DPPH • quanto o método $\beta$-caroteno avaliam a capacidade redutora das amostras [21,24].

Também foi encontrada uma alta correlação, acima de 0,70 , entre a atividade antioxidante avaliada pelos dois métodos e o teor de flavonóides e fenólicos totais, o que demonstra a importância dos flavonóides como agentes antioxidantes e com potencial na redução do risco de desenvolvimento de doenças crônicas.

Os principais compostos fenólicos da soja são as isoflavonas [9]. A concentração de isoflavonas na soja e de seus derivados pode variar muito, pois depende da variedade do grão, solo, clima, local onde foi cultivada e, principalmente, do tipo de processamento utilizado no preparo dos produtos protéicos [15]. As condições de processamento da soja podem provocar alterações, tanto no teor total como nas formas das isoflavonas presentes. Resultados anteriores mostraram que os teores de isoflavonas totais variam de 120 a $197 \mathrm{mg} / 100$ g para as farinhas desengorduradas, de 88 a $164 \mathrm{mg} / 100 \mathrm{~g}$

TABELA 3 - Determinação da capacidade antioxidante pelo seqüestro de radicais livres do DPPH $\bullet$, expressa em $\mu$ moles equivalentes de trolox/g amostra (b.s.), e pelo sistema $\beta$-caroteno/ácido linoléico, expressa em $\mu \mathrm{mol}$ BHT/g amostra (b.s.), da soja em grão, farinha desengordurada comercial, farinha integral, farinha desengordurada, isolado protéico, proteína texturizada e suplemento à base de gérmen de soja.

\begin{tabular}{|c|c|c|}
\hline Amostras & $\begin{array}{c}\mu \mathrm{mol} \mathrm{BHT} / \mathrm{g} \\
\text { amostra }\end{array}$ & $\begin{array}{c}\mu \text { moles eq. de trolox/g } \\
\text { amostra }\end{array}$ \\
\hline $\begin{array}{l}\text { Farinha desengordurada } \\
\text { de soja comercial }\end{array}$ & $0,75 \pm 0,02^{\mathrm{b}}$ & $4,2 \pm 0,6^{b}$ \\
\hline Farinha integral & $0,67 \pm 0,02^{c}$ & $4,0 \pm 0,4^{b}$ \\
\hline $\begin{array}{l}\text { Farinha desengordurada } \\
\text { de soja }\end{array}$ & $0,85 \pm 0,01^{a}$ & $5,0 \pm 0,1^{\mathrm{a}}$ \\
\hline Isolado protéico de soja & $0,58 \pm 0,01^{d}$ & $1,3 \pm 0,1^{c}$ \\
\hline $\begin{array}{l}\text { Proteína Texturizada } \\
\text { de soja }\end{array}$ & $0,73 \pm 0,02^{b}$ & $1,9 \pm 0,1^{\mathrm{d}}$ \\
\hline Grãos de soja & $0,72 \pm 0,03^{\mathrm{b}}$ & $3,7 \pm 0,3^{b}$ \\
\hline Suplemento & $0,78 \pm 0,03^{b}$ & $5,1 \pm 0,1^{a}$ \\
\hline
\end{tabular}

Os resultados estão expressos na forma de média \pm desvio padrão $(n=3)$. Médias com letras diferentes na mesma coluna são significativamente diferentes $(\mathrm{p}<0,05)$.

TABELA 2 - Distribuição de isoflavonas da soja em grão, farinha desengordurada comercial (FDS com.), farinha integral (FI), farinha desengordurada (FDS), isolado protéico (IPS), proteína texturizada (PTS) e suplemento à base de gérmen de soja.

\begin{tabular}{|c|c|c|c|c|c|c|c|}
\hline$(\%)^{*}$ & FDS com & FI & FDS & IPS & PTS & Grão & Suplemento \\
\hline$\beta$-glicosídeos & $50,4 \pm 0,4^{b}$ & $38,3 \pm 0,6^{d}$ & $39,1 \pm 0,6^{d}$ & $22,0 \pm 0,2^{\mathrm{e}}$ & $42,9 \pm 0,4^{c}$ & $42,8 \pm 0,1^{\mathrm{c}}$ & $78,6 \pm 0,5^{\mathrm{a}}$ \\
\hline Malonilglicosídeos & $40 \pm 1^{c}$ & $52,5 \pm 0,5^{a}$ & $50,6 \pm 0,6^{b}$ & $37,0 \pm 0,1^{\mathrm{d}}$ & $35,5 \pm 0,6^{\mathrm{e}}$ & $52,5 \pm 0,1^{a}$ & $8,6 \pm 0,2^{f}$ \\
\hline Acetilglicosídeos & $4,0 \pm 0,2^{\mathrm{c}}$ & $2,3 \pm 0,1^{d}$ & $2,9 \pm 0,1^{\mathrm{e}}$ & $4,1 \pm 0,1^{c}$ & $16,6 \pm 0,1^{a}$ & $1,0 \pm 0,1^{f}$ & $7 \pm 1^{b}$ \\
\hline Agliconas & $5,8 \pm 0,3^{c}$ & $6,7 \pm 0,4^{b}$ & $7,2 \pm 0,1^{b}$ & $36,8 \pm 0,1^{a}$ & $5,1 \pm 0,1^{d}$ & $4,0 \pm 0,1^{\mathrm{e}}$ & $6 \pm 1^{b, c, d}$ \\
\hline Daidzeína total & $38 \pm 1^{b}$ & $37 \pm 1^{b, c}$ & $35 \pm 2^{b, c}$ & $34 \pm 2^{c}$ & $38 \pm 1^{\mathrm{b}}$ & $39 \pm 1^{b}$ & $60 \pm 1^{a}$ \\
\hline Gliciteína total & $5 \pm 1^{c}$ & $6 \pm 1^{b, c}$ & $8 \pm 1^{b}$ & $4 \pm 1 c$ & $9 \pm 1^{b}$ & $7 \pm 1^{b}$ & $20 \pm 1^{a}$ \\
\hline Genisteína total & $57 \pm 1^{b}$ & $57 \pm 1^{\mathrm{b}}$ & $58 \pm 1^{b}$ & $62 \pm 1^{a}$ & $53 \pm 1^{c}$ & $54 \pm 1^{c}$ & $20 \pm 1^{d}$ \\
\hline
\end{tabular}

Os resultados estão expressos na forma de média \pm desvio padrão $(\mathrm{n}=3$ ). Médias na mesma linha com letras diferentes são significativamente diferentes ( $\mathrm{p}<0,05)$. ${ }^{*}$ As formas das isoflavonas (conjugados ou agliconas) foram expressas em\% do total (p/p). A porcentagem total de cada aglicona representa a soma das formas livres e conjugadas, expressas como agliconas, em relação ao total $(\mathrm{p} / \mathrm{p})$. 
para os isolados, de 194 a $727 \mathrm{mg} / 100$ g para hipocótilos (gérmen), e de 87 a 100 mg/100 g para texturizados de soja [8]. Os resultados aqui obtidos mostraram que os produtos analisados estão de acordo com os resultados anteriores. A distribuição entre as formas das isoflavonas varia de produto para produto (Tabela 2), e observa-se que a maioria (97$98 \%$ ) das isoflavonas em soja e derivados protéicos como farinhas, isolados, concentrados e proteína texturizada encontra-se na forma esterificada [27].

RUIZ-LARREA et al. [22] reportaram que a capacidade antioxidante das isoflavonas segue a ordem genisteína > daidzeína $>$ genistina $>$ daidzina, ou seja, está associada ao número de hidroxilas livres do anel A. Os $\beta$-glicosídeos, ao apresentarem $O$-glicosilação na posição 7 do anel A, apresentam redução na capacidade antioxidante. HASSIMOTTO et al. [13] também verificaram que a glicosilação afeta negativamente a capacidade antioxidante, já que a quercetina foi mais efetiva em inibir a oxidação do $\beta$-caroteno do que a rutina, sua forma glicosilada. Já nossos resultados mostraram que o suplemento à base de gérmen de soja foi o que apresentou maior capacidade antioxidante e tem como forma principal os $\beta$-glicosídeos, porém o conteúdo total das isoflavonas foi muito superior ao dos demais produtos. Por outro lado, o isolado protéico de soja, que apresentou maior teor de agliconas dentre as amostras analisadas (37\% do total), também apresentou a menor capacidade antioxidante pelos dois métodos, condizente com seu menor teor de fenólicos totais. Além disso, estima-se que, embora não haja relatos na literatura, também haja diferenças entre as atividades antioxidantes dos derivados malonilados, acetilados e desesterificados. Desta forma, a capacidade antioxidante dos derivados de soja resultaria não apenas dos teores de isoflavonas, mas sim de sua distribuição e formas presentes.

\section{4 - CONCLUSÕES}

Os resultados demonstraram que o conteúdo total e formas de isoflavonas e o teor de fenólicos dos produtos de soja dependem do processamento e que a capacidade antioxidante desses produtos varia significativamente, o que parece estar associado não apenas aos teores de compostos fenólicos totais, mas também à estrutura das agliconas e aos diferentes tipos de conjugação presentes.

\section{5 - REFERÊNCIAS BIBLIOGRÁFICAS}

[1] ASSOCIATION OF OFFICIAL ANALYTICAL CHEMISTS. Official Methods of Analysis of A. O. A. C. 16. ed. Washington, DC., 1995. 2000 p.

[2] BARBOSA, A. C. L.; LAJOLO, F. M.; GENOVESE, M. I. Influence of temperature, $\mathrm{pH}$ and ionic strength on the production of isoflavone-rich soy protein isolates. Food Chem., Oxford, v. 98, n. 4, p. 757-766, 2006.

[3] BRAND-WILliamS, W.; CUVELIER, M. E.; BERSET, C. Use of a free radical method to evaluate antioxidant activity. Food Sci. Technol., London, v. 28, n. 1, p. 25-30, 1995.
[4] COOK, N. S.; SAMMAN, S. Flavonoids - chemistry, metabolism, cadioprotective effect and dietary sources. J. Nutr. Biochem., New York, v. 7, n. 2, p. 66-76, 1996.

[5] COWARD, L. et al. Genistein, daidzein and their $\beta$-glycoside conjugates: antitumor isoflavones in soybean foods from American and Asian diets. J. Agric. Food Chem., Columbus, v. 41, n. 11, p. 1 961-1967, 1993.

[6] ELDRIDGE, A. C.; KWOLEK, W. F. Soybean isoflavones: effect of environment and variety on composition. J. Agric. Food Chem., Columbus, v. 31, n. 2, p. 394-396, 1983.

[7] FRITZ, K. L. et al. The in vivo antioxidant activity of soybean isoflavones in human subjects. Nutr. Res., New York, v. 23, n. 4, p. 479-487, 2003.

[8] GenoveSE, M. I.; LAJOLO, F. M. Determinação de isoflavonas em derivados de soja. Cienc. Tecnol. Alim., Campinas, v. 21, n. 1, p. 86-93, 2001.

[9] GENOVESE, M. I. HASSIMOTTO, N. M. A.; LAJOLO, F. M. Isoflavone profile and antioxidant activity of Brazilian soybean varieties. Food Sci. Technol. Int., London, v. 11, n. 3, p. 205-211, 2005.

[10] GENOVESE, M. I. et al. Determinação do conteúdo de fenólicos totais em frutos. Rev. Bras. Cienc. Farm. São Paulo, v. 39, supl. 3, p. 167-169, 2003.

[11] GRÜN, I. U. et al. Changes in the profile of genistein, daidzein, and their conjugates during thermal processing of tofu. J. Agric. Food Chem., Columbus, v. 49, n. 6, p. 2839-2843, 2001.

[12] HARBORNE, J. B.; WILLIANS, C. A. Advances in flavonoid research since 1992. Phytochem., Oxford, v. 55, n. 6, p. 481-504, 2000.

[13] HASsimotto, N. M. A.; GenOVESE, M. I.; LAJOLO, F.M. Antioxidant activity of dietary fruits, vegetables, and commercial frozen fruits pulps. J. Agric. Food Chem., Columbus, v. 53, n. 8, p. 2928-2935, 2005.

[14] KUDOU, D. et al. Malonyl isoflavone glycosides in soybean seeds (Glycine max Merrill). Agric. Biol. Chem., Tokyo, v. 55, n. 9, p. 2227-2233, 1991.

[15] LEE, S. J. et al. Effects of year, site, genotype and their interactions on various soybean isoflavones. Field Crops Res., Amsterdam, v. 81, n. 2/3, p. 181-192, 2003.

[16] LIU, J.; CHANG, S. K. C.; WIESENBORN, D. Antioxidant properties of soybean isoflavone extract and tofu in vitro and in vivo. J. Agric. Food Chem., Columbus, v. 53, n. 6, p. 2333-2340, 2005.

[17] MILLER, H. E. A simplified method for the evaluation of antioxidants. J. Am. Oil Chem. Soc., Champaing, v. 48, n. 2, p. $91,1971$.

[18] NAIM, M. et al. Antioxidative and antihemolytic activities of soybean isoflavones. J. Agric. Food Chem., Columbus, v. 24, n. 6, p. 1174-1177, 1976.

[19] PRATT, D. E.; BIRAC, P. M. Source of antioxidant activity of soybean and soy products. J.Food Sci., Chicago, v. 44, n. 6, p. 1720-1722, 1979.

[20] PRATT, D. E. et al. Inhibition of in vitro microsomal lipid peroxidation by isoflavonoids. J.Food Sci., Chicago, v. 47, n. 1 p. 24-25, 1981. 
[21] PRIOR, R. L.; WU, X.; SCHAICH, K. Standardized methods for the determination of antioxidant capacity and phenolics in foods and dietary supplements. J. Agric. Food Chem., Columbus, v. 53, n. 10, p. 4290-4302, 2005.

[22] RUIZ-LARREA, M. B. et al. Antioxidant activity of phytoestrogenic isoflavones. Free Rad. Res., London, v. 26, n. 1, p. 63-70, 1997.

[23] SETCHELL, K. D. R.; CASSIDY, A. Dietary isoflavones: biological effects and relevance to human health. J. Nutr., Bethesda, v. 129, n. 3, p. 758S-767S, 1999.

[24] SILVA, F. A. M.; BORGES, M. F. M.; FERREIRA, M. A. Método para avaliação do grau de oxidação lipídica e da capacidade antioxidante. Quim. Nova, São Paulo, v. 22, n. 1, p. 94-103, 1999.

[25] SONG, T. et al. Soy isoflavones analysis: quality control and a new internal standard. Am. J. Clin. Nutr., Bethesda, v. 68, supp. 4, p. 1474S-1479S, 1998.

[26] THAM, D. M.; GARDNER, C. D.; HASKELL, W. L. Clinical review 97: Potential health benefits of dietary phytoestrogens. J. Clin. Endocrinol. Metab., Chevy Chase, MD., v. 83, n. 7, p. 2223-2235, 1998.

[27] WANG, H.; MURPHY, P. A. Isoflavone content in commercial soybean foods. J. Agric. Food Chem., Columbus, v. 42, n. 8, p. 1666-1673, 1994.

[28] YEN, G. C.; LAI, H. H. Inhibition of reactive nitrogen species effects in vitro and in vivo by isoflavones and soy-based food extracts. J. Agric. Food Chem., Columbus, v. 51, n. 27, p. 7892-7900, 2003.

[29] ZHANG, X. et al. Soy food consumption is associated with lower risk of coronary heart disease in Chinese women. J. Nutr., Bethesda, v. 133, n. 9, p. 2874-2878, 2003.

[30] ZIELISKI, H.; KOZOWSKA, H. Antioxidant activity and total phenolics in selected cereal grains and their different morphological fractions. J. Agric. Food Chem. Columbus, v. 48, n. 6, p. 2008-2016, 2000.

\section{6 - AGRADECIMENTOS}

À FAPESP, CAPES e CNPq pelo auxílio financeiro. 